\title{
Algebraic integers as values of elliptic functions
}

by

\author{
Daeyeoul Kim (Chonju) and Ja Kyung Koo (Taejon)
}

0. Introduction. In this paper, we shall deal with certain algebraic integers as values of elliptic functions constructed from the Weierstrass $\wp$ function by using infinite products (Theorem 2.2). In the process we are able to reprove the well known fact that $j(\tau)$ is an algebraic integer for an imaginary quadratic $\tau$; our proof seems to be quite simple and elementary unlike the others ([3]-[8]). And in Section 3 we shall derive analogues (Theorem 3.2) of Berndt-Chan-Zhang's results, which could be a generalization in the case of $m$ even. In the last section, we explore some algebraic properties of values of the Weierstrass $\wp$-function and Fricke functions.

1. Infinite product formulas for the Weierstrass $\wp$-function. Let $\Lambda_{\tau}=\mathbb{Z}+\tau \mathbb{Z}(\tau \in \mathfrak{h})$ be a lattice and $z \in \mathbb{C}$. The Weierstrass $\wp$-function (relative to $\Lambda_{\tau}$ ) is defined by the series

$$
\wp\left(z ; \Lambda_{\tau}\right)=\frac{1}{z^{2}}+\sum_{\substack{\omega \in \Lambda_{\tau} \\ \omega \neq 0}}\left\{\frac{1}{(z-\omega)^{2}}-\frac{1}{\omega^{2}}\right\},
$$

and the Eisenstein series of weight $2 k$ (for $\Lambda_{\tau}$ and $k>1$ ) is the series

$$
G_{2 k}\left(\Lambda_{\tau}\right)=\sum_{\substack{\omega \in \Lambda_{\tau} \\ \omega \neq 0}} \omega^{-2 k}
$$

We shall use the notations $\wp(z)$ and $G_{2 k}$ instead of $\wp\left(z ; \Lambda_{\tau}\right)$ and $G_{2 k}\left(\Lambda_{\tau}\right)$, respectively, when the lattice $\Lambda_{\tau}$ has been fixed.

Then the Laurent series for $\wp(z)$ about $z=0$ is given by

$$
\wp(z)=z^{-2}+\sum_{k=1}^{\infty}(2 k+1) G_{2 k+2} z^{2 k}
$$

2000 Mathematics Subject Classification: 11J89, 05A30.

Key words and phrases: infinite product, algebraic integer.

This article was supported in part by KOSEF 98-0701-01-01-3. 
and, for all $z \in \mathbb{C}-\Lambda_{\tau}$ we obtain the equation

$$
\wp^{\prime}(z)^{2}=4 \wp(z)^{3}-60 G_{4} \wp(z)-140 G_{6} .
$$

As is customary, the algebraic relation between $\wp(z)$ and $\wp^{\prime}(z)$ becomes

$$
\wp^{\prime}(z)^{2}=4 \wp(z)^{3}-g_{2}(\tau) \wp(z)-g_{3}(\tau),
$$

where

$$
g_{2}(\tau)=g_{2}\left(\Lambda_{\tau}\right)=60 G_{4} \quad \text { and } \quad g_{3}(\tau)=g_{3}\left(\Lambda_{\tau}\right)=140 G_{6} .
$$

Moreover, we have the following proposition at hand which will be useful in extracting infinite product expressions.

Proposition 1.1 ([4], [8]). Let $p=e^{\pi i \tau}$.

$$
\begin{aligned}
& \text { (1) } \wp\left(\frac{\tau}{2}\right)-\wp\left(\frac{1}{2}\right)=-\pi^{2} \prod_{n=1}^{\infty}\left(1-p^{2 n}\right)^{4}\left(1+p^{2 n-1}\right)^{8} . \\
& \text { (2) } \wp\left(\frac{\tau+1}{2}\right)-\wp\left(\frac{1}{2}\right)=-\pi^{2} \prod_{n=1}^{\infty}\left(1-p^{2 n}\right)^{4}\left(1-p^{2 n-1}\right)^{8} . \\
& \text { (3) } \wp\left(\frac{\tau+1}{2}\right)-\wp\left(\frac{\tau}{2}\right)=16 \pi^{2} p \prod_{n=1}^{\infty}\left(1-p^{2 n}\right)^{4}\left(1+p^{2 n}\right)^{8} .
\end{aligned}
$$

Now, for simplicity we set

$$
\begin{array}{lll}
C:=\prod_{n=1}^{\infty}\left(1-p^{n}\right), & D:=\prod_{n=1}^{\infty}\left(1+p^{n}\right), & S:=\prod_{n=1}^{\infty}\left(1-p^{2 n}\right), \\
T:=\prod_{n=1}^{\infty}\left(1+p^{2 n-1}\right), & U:=\prod_{n=1}^{\infty}\left(1+p^{2 n}\right), & V:=\prod_{n=1}^{\infty}\left(1-p^{2 n-1}\right) .
\end{array}
$$

We then readily check that

$$
C D=S, \quad T U=D, \quad S U V T=S, \quad V U T=1 .
$$

By definition

$$
\Delta(\tau)=(2 \pi)^{12} \eta(\tau)^{24}=g_{2}(\tau)^{3}-27 g_{3}(\tau)^{2},
$$

which is the discriminant of the cubic polynomial

$$
4 \wp(z)^{3}-g_{2}(\tau) \wp(z)-g_{3}(\tau)=4\left(\wp(z)-e_{1}\right)\left(\wp(z)-e_{2}\right)\left(\wp(z)-e_{3}\right) .
$$

On the other hand, we know $([9])$ that the roots of this polynomial are

$$
e_{1}=\wp\left(\frac{1}{2}\right), \quad e_{2}=\wp\left(\frac{\tau}{2}\right), \quad e_{3}=\wp\left(\frac{\tau+1}{2}\right) .
$$

Thus, we have

$$
\wp\left(\frac{1}{2}\right)+\wp\left(\frac{\tau}{2}\right)+\wp\left(\frac{\tau+1}{2}\right)=0
$$




$$
\wp\left(\frac{1}{2}\right) \wp\left(\frac{\tau}{2}\right)+\wp\left(\frac{\tau+1}{2}\right) \wp\left(\frac{\tau}{2}\right)+\wp\left(\frac{1}{2}\right) \wp\left(\frac{\tau+1}{2}\right)=-\frac{g_{2}(\tau)}{4}
$$

and

$$
\wp\left(\frac{1}{2}\right) \wp\left(\frac{\tau}{2}\right) \wp\left(\frac{\tau+1}{2}\right)=\frac{g_{3}(\tau)}{4} .
$$

By the above equations and Proposition 1.1, we derive that

$$
\begin{aligned}
2 \wp\left(\frac{\tau}{2}\right)+\wp\left(\frac{\tau+1}{2}\right) & =\wp\left(\frac{\tau}{2}\right)-\left(-\wp\left(\frac{\tau+1}{2}\right)-\wp\left(\frac{\tau}{2}\right)\right) \\
& =\wp\left(\frac{\tau}{2}\right)-\wp\left(\frac{1}{2}\right)=-\pi^{2} S^{4} T^{8} .
\end{aligned}
$$

And we get the following three new identities:

$$
\begin{aligned}
& \wp\left(\frac{\tau}{2}\right)= \frac{1}{3}\left[\left(2 \wp\left(\frac{\tau}{2}\right)+\wp\left(\frac{\tau+1}{2}\right)\right)+\left(\wp\left(\frac{\tau}{2}\right)-\wp\left(\frac{\tau+1}{2}\right)\right)\right] \\
&=-\frac{\pi^{2}}{3} S^{4}\left(T^{8}+16 p U^{8}\right) \\
&=-\frac{\pi^{2}}{3} \prod_{n=1}^{\infty}\left(1-p^{2 n}\right)^{4} \\
& \times\left(\prod_{n=1}^{\infty}\left(1+p^{2 n-1}\right)^{8}+16 p \prod_{n=1}^{\infty}\left(1+p^{2 n}\right)^{8}\right), \\
& \wp\left(\frac{\tau+1}{2}\right)=16 \pi^{2} p S^{4} U^{8}-\frac{\pi^{2}}{3} S^{4}\left(T^{8}+16 p U^{8}\right) \\
&=-\frac{\pi^{2}}{3} S^{4}\left(T^{8}-32 p U^{8}\right) \\
&=-\frac{\pi^{2}}{3} \prod_{n=1}^{\infty}\left(1-p^{2 n}\right)^{4} \\
& \times\left(\prod_{n=1}^{\infty}\left(1+p^{2 n-1}\right)^{8}-32 p \prod_{n=1}^{\infty}\left(1+p^{2 n}\right)^{8}\right)
\end{aligned}
$$

and

(1.3)

$$
\begin{aligned}
\wp\left(\frac{1}{2}\right)= & \pi^{2} S^{4} T^{8}-\frac{\pi^{2}}{3} S^{4}\left(T^{8}+16 p U^{8}\right)=\frac{\pi^{2}}{3} S^{4}\left(2 T^{8}-16 p U^{8}\right) \\
= & \frac{\pi^{2}}{3} \prod_{n=1}^{\infty}\left(1-p^{2 n}\right)^{4} \\
& \times\left(2 \prod_{n=1}^{\infty}\left(1+p^{2 n-1}\right)^{8}-16 p \prod_{n=1}^{\infty}\left(1+p^{2 n}\right)^{8}\right) .
\end{aligned}
$$


Using (1.1)-(1.3) we obtain the identity for $g_{2}(\tau)$ :

$$
\begin{aligned}
g_{2}(\tau)= & -4\left[\wp\left(\frac{1}{2}\right) \wp\left(\frac{\tau}{2}\right)+\wp\left(\frac{\tau+1}{2}\right) \wp\left(\frac{\tau}{2}\right)+\wp\left(\frac{1}{2}\right) \wp\left(\frac{\tau+1}{2}\right)\right] \\
= & -4\left[\frac{\pi^{2}}{3} S^{4}\left(2 T^{8}-16 p U^{8}\right)\left(-\frac{\pi^{2}}{3} S^{4}\left(T^{8}+16 p U^{8}\right)\right)\right. \\
& +\left(-\frac{\pi^{2}}{3} S^{4}\left(T^{8}-32 p U^{8}\right)\right)\left(-\frac{\pi^{2}}{3} S^{4}\left(T^{8}+16 p U^{8}\right)\right) \\
& \left.+\left(\frac{\pi^{2}}{3} S^{4}\left(2 T^{8}-16 p U^{8}\right)\right)\left(-\frac{\pi^{2}}{3} S^{4}\left(T^{8}-32 p U^{8}\right)\right)\right] \\
= & \frac{4 \pi^{4}}{3} S^{8}\left(T^{16}-16 p T^{8} U^{8}+256 p^{2} U^{16}\right) \\
= & \frac{4 \pi^{4}}{3} \prod_{n=1}^{\infty}\left(1-p^{2 n}\right)^{8}\left(\prod_{n=1}^{\infty}\left(1+p^{2 n-1}\right)^{16}\right. \\
& \left.-16 p \prod_{n=1}^{\infty}\left(1+p^{n}\right)^{8}+256 p^{2} \prod_{n=1}^{\infty}\left(1+p^{2 n}\right)^{16}\right) .
\end{aligned}
$$

We are also able to express $g_{3}(\tau)$ as

$$
\begin{aligned}
g_{3}(\tau)= & 4 \wp\left(\frac{1}{2}\right) \wp\left(\frac{\tau}{2}\right) \wp\left(\frac{\tau+1}{2}\right) \\
= & 4\left(\frac{\pi^{2}}{3} S^{4}\left(2 T^{8}-16 p U^{8}\right)\right) \\
& \times\left(-\frac{\pi^{2}}{3} S^{4}\left(T^{8}-32 p U^{8}\right)\right)\left(-\frac{\pi^{2}}{3} S^{4}\left(T^{8}+16 p U^{8}\right)\right) \\
= & \frac{8 \pi^{6}}{27} S^{12}\left(T^{24}-24 p T^{16} U^{8}-384 p^{2} T^{8} U^{16}+4096 p^{3} U^{24}\right) \\
= & \frac{8 \pi^{6}}{27} \prod_{n=1}^{\infty}\left(1-p^{2 n}\right)^{12} \\
& \times\left(\prod_{n=1}^{\infty}\left(1+p^{2 n-1}\right)^{24}-24 p \prod_{n=1}^{\infty}\left(1+p^{2 n-1}\right)^{16}\left(1+p^{2 n}\right)^{8}\right. \\
& -384 p^{2} \prod_{n=1}^{\infty}\left(1+p^{2 n-1}\right)^{8}\left(1+p^{2 n}\right)^{16} \\
& \left.+4096 p^{3} \prod_{n=1}^{\infty}\left(1+p^{2 n}\right)^{24}\right) .
\end{aligned}
$$


Then, we derive from (1.4) and (1.5) that

$$
\begin{aligned}
j(\tau) & =1728 \frac{g_{2}(\tau)^{3}}{\Delta(\tau)} \\
& =1728 \frac{\left[\frac{4}{3} \pi^{4} S^{8}\left(T^{16}-16 p D^{8}+256 p^{2} U^{16}\right)\right]^{3}}{(2 \pi)^{12} p^{2} S^{24}} \\
& =\frac{1}{p^{2}}\left(T^{16}-16 p D^{8}+256 p^{2} U^{16}\right)^{3} .
\end{aligned}
$$

2. Some infinite products as algebraic integers. Throughout Sections 2 to 4 we shall fix the following notations: $k$ is an imaginary quadratic field, $\mathfrak{h}$ the complex upper half plane and $\tau \in \mathfrak{h} \cap k$.

Let $\alpha=\left(\begin{array}{ll}a & b \\ 0 & d\end{array}\right)$ with $b \bmod d$ and $|\alpha|$ the determinant of $\alpha$, and let

$$
\phi_{\alpha}(\tau):=|\alpha|^{12} \frac{\Delta\left(\alpha\left(\begin{array}{c}
\tau \\
1
\end{array}\right)\right)}{\Delta\left(\left(\begin{array}{c}
\tau \\
1
\end{array}\right)\right)}=|\alpha|^{12} d^{-12} \frac{\Delta(\alpha \tau)}{\Delta(\tau)} .
$$

Then we recall the following well known fact.

Proposition 2.1 ([4]). For any $\tau \in k \cap \mathfrak{h}$, the value $\phi_{\alpha}(\tau)$ is an algebraic integer which divides $|\alpha|^{12}$.

First, we consider

$$
\frac{\Delta(\tau)}{\Delta(\tau / 2)}=\frac{(2 \pi)^{12} p^{2} \prod_{n=1}^{\infty}\left(1-p^{2 n}\right)^{24}}{(2 \pi)^{12} p \prod_{n=1}^{\infty}\left(1-p^{n}\right)^{24}}=p \prod_{n=1}^{\infty}\left(1+p^{n}\right)^{24}
$$

and

$$
\frac{\Delta(\tau / 2)}{\Delta(\tau)}=\frac{(2 \pi)^{12} p \prod_{n=1}^{\infty}\left(1-p^{n}\right)^{24}}{(2 \pi)^{12} p^{2} \prod_{n=1}^{\infty}\left(1-p^{2 n}\right)^{24}}=p^{-1} \frac{1}{\prod_{n=1}^{\infty}\left(1+p^{n}\right)^{24}} .
$$

Put

$$
\alpha_{1}=\left(\begin{array}{cc}
2 & 0 \\
0 & 1
\end{array}\right), \quad \alpha_{2}=\left(\begin{array}{ll}
1 & 0 \\
0 & 2
\end{array}\right)
$$

By $(*)$,

$$
\phi_{\alpha_{1}}(\tau / 2)=2^{12} \frac{\Delta(\tau)}{\Delta(\tau / 2)} \quad\left(=2^{12} \frac{\eta(\tau)^{24}}{\eta(\tau / 2)^{24}}\right),
$$

from which we see by Proposition 2.1 that

$$
\sqrt{2} p^{1 / 24} \prod_{n=1}^{\infty}\left(1+p^{n}\right)
$$

is an algebraic integer. Also, we have

$$
\phi_{\alpha_{2}}(\tau)=2^{12} \frac{1}{2^{12}} \cdot \frac{\Delta(\tau / 2)}{\Delta(\tau)} \quad\left(=\frac{\eta(\tau / 2)^{24}}{\eta(\tau)^{24}}\right),
$$


and hence

$$
p^{-1 / 24} \frac{1}{\prod_{n=1}^{\infty}\left(1+p^{n}\right)}
$$

is an algebraic integer.

It follows from (1.0) that

$$
\prod_{n=1}^{\infty}\left(1+p^{2 n}\right)\left(1+p^{2 n-1}\right)\left(1-p^{2 n-1}\right)=1 .
$$

So, by (2.2) and the above, we obtain

$$
\begin{aligned}
\frac{1}{p^{2} \prod_{n=1}^{\infty}\left(1+p^{2 n}\right)^{24}} & =\frac{\prod_{n=1}^{\infty}\left(1+p^{2 n}\right)^{24}\left(1+p^{2 n-1}\right)^{24}\left(1-p^{2 n-1}\right)^{24}}{p^{2} \prod_{n=1}^{\infty}\left(1+p^{2 n}\right)^{24}} \\
& =p^{-2} \prod_{n=1}^{\infty}\left(1-p^{4 n-2}\right)^{24}
\end{aligned}
$$

Thus we see from (2.2) that

$$
p^{-1 / 24} \prod_{n=1}^{\infty}\left(1-p^{2 n-1}\right)
$$

is an algebraic integer. By (2.1) and (2.3), we claim that

$$
\sqrt{2} \prod_{n=1}^{\infty}\left(1+p^{n}\right)\left(1-p^{2 n-1}\right)
$$

is an algebraic integer.

Jacobi ([10, p. 470]) showed that

$$
\prod_{n=1}^{\infty}\left(1+p^{2 n-1}\right)^{8}-\prod_{n=1}^{\infty}\left(1-p^{2 n-1}\right)^{8}=16 p \prod_{n=1}^{\infty}\left(1+p^{2 n}\right)^{8},
$$

which we can now easily check by using Proposition 1.1.

Multiplying both sides in Jacobi's relation by $p^{-1 / 3}$, we derive from (2.1) and (2.3) that

$$
p^{-1 / 24} \prod_{n=1}^{\infty}\left(1+p^{2 n-1}\right)
$$

is an algebraic integer.

Combining (2.1) and (2.5) we see that

$$
\sqrt{2} \prod_{n=1}^{\infty}\left(1+p^{n}\right)\left(1+p^{2 n-1}\right)
$$

is also an algebraic integer. 
By (1.6), (2.1) and (2.5) we are led to

$$
\begin{aligned}
j(\tau)^{1 / 3}= & -16 p^{1 / 3} \prod_{n=1}^{\infty}\left(1+p^{n}\right)^{8}+256 p^{4 / 3} \prod_{n=1}^{\infty}\left(1+p^{2 n}\right)^{16} \\
& +p^{-2 / 3} \prod_{n=1}^{\infty}\left(1+p^{2 n-1}\right)^{16}
\end{aligned}
$$

from which we can reprove the well known fact ([3]-[8]) that $j(\tau)$ is an algebraic integer. Observe that, in the above, we used the fact that $\sqrt{2} p^{1 / 12} \prod_{n=1}^{\infty}\left(1+p^{2 n}\right)$ is also an algebraic integer, which can be readily deduced from (2.1).

On the other hand, we know by (1.1) that

$$
\begin{aligned}
\frac{-3 \wp(\tau / 2)}{\pi^{2} \eta(\tau)^{4}} & =\frac{\prod_{n=1}^{\infty}\left(1-p^{2 n}\right)^{4}\left(\left(1+p^{2 n-1}\right)^{8}+16 p\left(1+p^{2 n}\right)^{8}\right)}{p^{1 / 3} \prod_{n=1}^{\infty}\left(1-p^{2 n}\right)^{4}} \\
& =p^{-1 / 3} \prod_{n=1}^{\infty}\left(1+p^{2 n-1}\right)^{8}+16 p^{2 / 3} \prod_{n=1}^{\infty}\left(1+p^{2 n}\right)^{8} .
\end{aligned}
$$

We then conclude from (2.1) and (2.5) that

$$
\frac{3}{\pi^{2}} \cdot \frac{\wp(\tau / 2)}{\eta(\tau)^{4}}
$$

is an algebraic integer. Also, it follows from (1.4) that

$$
\begin{aligned}
\frac{3 g_{2}(\tau)}{4 \pi^{4} \eta(\tau)^{8}}= & \frac{\prod_{n=1}^{\infty}\left(1-p^{2 n}\right)^{8}\left(1+p^{2 n-1}\right)^{16}-16 p \prod_{n=1}^{\infty}\left(1-p^{2 n}\right)^{8}\left(1+p^{n}\right)^{8}}{p^{2 / 3} \prod_{n=1}^{\infty}\left(1-p^{2 n}\right)^{8}} \\
& +\frac{256 p^{2} \prod_{n=1}^{\infty}\left(1-p^{2 n}\right)^{8}\left(1+p^{2 n}\right)^{16}}{p^{2 / 3} \prod_{n=1}^{\infty}\left(1-p^{2 n}\right)^{8}} \\
= & p^{-2 / 3} \prod_{n=1}^{\infty}\left(1+p^{2 n-1}\right)^{16}-16 p^{1 / 3} \prod_{n=1}^{\infty}\left(1+p^{n}\right)^{8} \\
& +256 p^{4 / 3} \prod_{n=1}^{\infty}\left(1+p^{2 n}\right)^{16} .
\end{aligned}
$$

Thus we find again by (2.1) and (2.5) that

$$
\frac{3}{4 \pi^{4}} \cdot \frac{g_{2}(\tau)}{\eta(\tau)^{8}}
$$

is an algebraic integer. And we deduce from (1.5) that 


$$
\begin{aligned}
\frac{27 g_{3}(\tau)}{\pi^{6} \eta(\tau)^{12}}= & 8 p^{-1} \prod_{n=1}^{\infty}\left(1+p^{2 n-1}\right)^{24}-192 \prod_{n=1}^{\infty}\left(1+p^{n}\right)^{8}\left(1+p^{2 n-1}\right)^{8} \\
& -3072 p \prod_{n=1}^{\infty}\left(1+p^{n}\right)^{8}\left(1+p^{2 n}\right)^{8}+32768 p^{2} \prod_{n=1}^{\infty}\left(1+p^{2 n}\right)^{24}
\end{aligned}
$$

from which we conclude by $(2.1),(2.5)$ and (2.6) that

$$
\frac{27}{\pi^{6}} \cdot \frac{g_{3}(\tau)}{\eta(\tau)^{12}}
$$

is an algebraic integer.

By Proposition 1.1, (2.1), (2.3) and (2.5), we derive that

$$
\frac{\wp\left(\frac{\tau}{2}\right)-\wp\left(\frac{1}{2}\right)}{\pi^{2} \eta(\tau)^{4}}, \quad \frac{\wp\left(\frac{\tau+1}{2}\right)-\wp\left(\frac{1}{2}\right)}{\pi^{2} \eta(\tau)^{4}} \text { and } \quad \frac{\wp\left(\frac{\tau+1}{2}\right)-\wp\left(\frac{\tau}{2}\right)}{\pi^{2} \eta(\tau)^{4}}
$$

are algebraic integers. Also, it follows from (2.8) and (2.11) that

$$
\frac{3}{\pi^{2}} \cdot \frac{\wp((\tau+1) / 2)}{\eta(\tau)^{4}} \text { and } \frac{3}{\pi^{2}} \cdot \frac{\wp(1 / 2)}{\eta(\tau)^{4}}
$$

are algebraic integers.

We summarize (2.1) to (2.12) as follows.

TheOREM 2.2. Let $\tau \in k \cap \mathfrak{h}$. Then

$$
\begin{aligned}
\text { (a) } & \sqrt{2} p^{1 / 24} \prod_{n=1}^{\infty}\left(1+p^{n}\right), \quad p^{-1 / 24} \frac{1}{\prod_{n=1}^{\infty}\left(1+p^{n}\right)}, \quad p^{-1 / 24} \prod_{n=1}^{\infty}\left(1-p^{2 n-1}\right), \\
& \sqrt{2} \prod_{n=1}^{\infty}\left(1+p^{n}\right)\left(1-p^{2 n-1}\right), \quad p^{-1 / 24} \prod_{n=1}^{\infty}\left(1+p^{2 n-1}\right) \quad \text { and } \\
& \sqrt{2} \prod_{n=1}^{\infty}\left(1+p^{n}\right)\left(1+p^{2 n-1}\right)
\end{aligned}
$$

are algebraic integers.

$$
\begin{aligned}
& \text { (b) } j(\tau), \quad \frac{3}{\pi^{2}} \cdot \frac{\wp\left(\frac{\tau}{2}\right)}{\eta(\tau)^{4}}, \quad \frac{3}{\pi^{2}} \cdot \frac{\wp\left(\frac{\tau+1}{2}\right)}{\eta(\tau)^{4}}, \quad \frac{3}{\pi^{2}} \cdot \frac{\wp\left(\frac{1}{2}\right)}{\eta(\tau)^{4}}, \quad \frac{3}{4 \pi^{4}} \cdot \frac{g_{2}(\tau)}{\eta(\tau)^{8}}, \\
& \frac{27}{\pi^{6}} \cdot \frac{g_{3}(\tau)}{\eta(\tau)^{12}}, \quad \frac{\wp\left(\frac{\tau}{2}\right)-\wp\left(\frac{1}{2}\right)}{\pi^{2} \eta(\tau)^{4}}, \quad \frac{\wp\left(\frac{\tau+1}{2}\right)-\wp\left(\frac{1}{2}\right)}{\pi^{2} \eta(\tau)^{4}} \text { and } \\
& \frac{\wp\left(\frac{\tau+1}{2}\right)-\wp\left(\frac{\tau}{2}\right)}{\pi^{2} \eta(\tau)^{4}}
\end{aligned}
$$

are algebraic integers.

The Gel'fond-Schneider theorem says that $e^{\pi \alpha}=(-1)^{-i \alpha}$ is transcendental whenever $i \alpha$ is algebraic of degree at least 2 over $\mathbb{Q}([8]$, p. 142). This facts yields that $p=e^{\pi i \tau}$ is transcendental. Therefore, we have 
COROllary 2.3. Let $\tau \in k \cap \mathfrak{h}$. Then

$$
\prod_{n=1}^{\infty}\left(1+p^{n}\right), \quad \prod_{n=1}^{\infty}\left(1-p^{2 n-1}\right) \quad \text { and } \quad \prod_{n=1}^{\infty}\left(1+p^{2 n-1}\right)
$$

are transcendental numbers.

3. Approach to $\phi(\tau)$. Let

$$
\phi(\tau):=\phi\left(e^{\pi i \tau}\right)=\frac{\eta((\tau+1) / 2)^{2}}{\eta(\tau+1)}=\prod_{n=1}^{\infty}\left(1+p^{2 n-1}\right)^{2}\left(1-p^{2 n}\right)=\theta_{3}(0, \tau) .
$$

Here we refer to [2] for the last equality. Berndt, Chan and Zhang showed in [1] the following proposition by using three of Ramanujan's modular equations, values of certain class invariants of Ramanujan, representations for quotients of values of $\phi$ in terms of class invariants and the thetatransformation formula.

In this section we shall derive certain analogues of their results purely in terms of infinite products, which is a generalization in the case of $m$ even.

Proposition 3.1. Let $m$ and $n$ be positive integers. Then $\phi(m n i) / \phi(n i)$ is algebraic. Furthermore, if $m$ is odd, then $\sqrt{2 m} \phi(m n i) / \phi(n i)$ is an algebraic integer dividing $2 \sqrt{m}$, while if $m$ is even, then $2 \sqrt{m} \phi(m n i) / \phi(n i)$ is an algebraic integer dividing $4 \sqrt{m}$.

By (2.5), the value

$$
\frac{\phi(\tau)}{\eta(\tau)}=\frac{\prod_{n=1}^{\infty}\left(1+p^{2 n-1}\right)^{2}\left(1-p^{2 n}\right)}{p^{1 / 12} \prod_{n=1}^{\infty}\left(1-p^{2 n}\right)}=p^{-1 / 12} \prod_{n=1}^{\infty}\left(1+p^{2 n-1}\right)^{2}
$$

is an algebraic integer. Observe that it can also be written as $\theta_{3}(0, \tau) / \eta(\tau)$.

By considering the identity $V U T=1$ from (1.0), that is,

$$
\prod_{n=1}^{\infty}\left(1+p^{2 n}\right)\left(1+p^{2 n-1}\right)\left(1-p^{2 n-1}\right)=1
$$

we have

$$
\begin{aligned}
\frac{\eta(\tau)}{\phi(\tau)} & =p^{1 / 12} \prod_{n=1}^{\infty}\left(1-p^{2 n}\right) \frac{1}{\prod_{n=1}^{\infty}\left(1+p^{2 n-1}\right)^{2}\left(1-p^{2 n}\right)} \\
& =p^{1 / 12} \prod_{n=1}^{\infty}\left(1-p^{2 n}\right) \frac{\prod_{n=1}^{\infty}\left(1+p^{2 n}\right)^{2}\left(1+p^{2 n-1}\right)^{2}\left(1-p^{2 n-1}\right)^{2}}{\prod_{n=1}^{\infty}\left(1+p^{2 n-1}\right)^{2}\left(1-p^{2 n}\right)} \\
& =p^{1 / 12} \prod_{n=1}^{\infty}\left(1+p^{2 n}\right)^{2}\left(1-p^{2 n-1}\right)^{2} .
\end{aligned}
$$


Thus the value

$$
2 \frac{\eta(\tau)}{\phi(\tau)}
$$

is an algebraic integer by (2.1) and (2.3).

Let $r, s, u, v$ be positive integers with $(r, s)=(u, v)=1$. Then $\frac{r}{s} \tau$ and $\frac{u}{v} \tau$ are still imaginary quadratic.

By (3.1) and (3.2) we see that

$$
\frac{\phi\left(\frac{s}{r} \tau\right)}{\eta\left(\frac{s}{r} \tau\right)} \cdot 2 \frac{\eta(\tau)}{\phi(\tau)}=2 \frac{\phi\left(\frac{s}{r} \tau\right)}{\phi(\tau)} \cdot \frac{\eta(\tau)}{\eta\left(\frac{s}{r} \tau\right)}
$$

and

$$
2 \frac{\phi(\tau)}{\phi\left(\frac{u}{v} \tau\right)} \cdot \frac{\eta\left(\frac{u}{v} \tau\right)}{\eta(\tau)}
$$

are algebraic integers.

Let

$$
\alpha_{r / s}=\left(\begin{array}{cc}
r & 0 \\
0 & s
\end{array}\right), \quad \alpha_{v / u}=\left(\begin{array}{cc}
v & 0 \\
0 & u
\end{array}\right) .
$$

Then by the above and Proposition 2.1 we derive that

$$
2 \frac{\phi\left(\frac{r}{s} \tau\right)}{\phi(\tau)} \cdot \frac{\eta(\tau)}{\eta\left(\frac{r}{s} \tau\right)} \cdot \phi_{\alpha_{r / s}}(\tau)^{1 / 24}=2 \sqrt{r} \frac{\phi\left(\frac{r}{s} \tau\right)}{\phi(\tau)}
$$

and

$$
2 \frac{\phi(\tau)}{\phi\left(\frac{u}{v} \tau\right)} \cdot \frac{\eta\left(\frac{u}{v} \tau\right)}{\eta(\tau)} \cdot \phi_{\alpha_{v} / u}\left(\frac{u}{v} \tau\right)^{1 / 24}=2 \sqrt{v} \frac{\phi(\tau)}{\phi\left(\frac{u}{v} \tau\right)}
$$

are algebraic integers.

Therefore, we have the following theorem.

TheOREM 3.2. Let $\tau$ be any imaginary quadratic and $r, s, u, v$ be positive integers such that $(r, s)=(u, v)=1$. Then $4 \sqrt{r v} \phi\left(\frac{r}{s} \tau\right) / \phi\left(\frac{u}{v} \tau\right)$ is an algebraic integer dividing $\sqrt{r s u v}$. In particular, $2 \sqrt{r} \phi\left(\frac{r}{s} \cdot \frac{u}{v} \tau\right) / \phi\left(\frac{u}{v} \tau\right)$ and $2 \sqrt{v} \phi\left(\frac{r}{s} \tau\right) / \phi\left(\frac{u}{v} \cdot \frac{r}{s} \tau\right)$ are algebraic integers dividing $\sqrt{r s}$ and $\sqrt{u v}$, respectively.

REMARK 3.3. Theorem 3.2 ensures that, in fact, the algebraic integer $\sqrt{2 m} \phi(m n i) / \phi(n i)$ (respectively, $2 \sqrt{m} \phi(m n i) / \phi(n i))$ in Proposition 3.1 divides $\sqrt{m / 2}$ (resp., $\sqrt{m}$ ) when $m$ is odd (resp., even).

In a similar way, when working with the matrices $\left(\begin{array}{ll}1 & j \\ 0 & n\end{array}\right)(0 \leq j \leq n-1)$, we derive that $\phi\left(\frac{\tau}{n}\right) / \phi(\tau), \ldots, \phi\left(\frac{\tau+n-1}{n}\right) / \phi(\tau)$ are algebraic numbers; hence $\phi\left(\frac{\tau}{n}\right) \ldots \phi\left(\frac{\tau+n-1}{n}\right) / \phi(\tau)^{n}$ is an algebraic number. This implies that there 
exists a polynomial $f(X)=a_{0} X^{l}+a_{1} X^{l-1}+\ldots+a_{l}$ in $\mathbb{Q}[X]$ satisfying

$$
f\left(\frac{\phi\left(\frac{\tau}{n}\right) \ldots \phi\left(\frac{\tau+n-1}{n}\right)}{\phi(\tau)^{n}}\right)=0 .
$$

Therefore we get an equation

$$
a_{l} \phi(\tau)^{n l}+\ldots+a_{0}\left(\phi\left(\frac{\tau}{n}\right) \ldots \phi\left(\frac{\tau+n-1}{n}\right)\right)^{l}=0
$$

which leads us to the following

TheOREM 3.4. Let $n$ be any positive integer. Then $\phi(\tau)$ is integral over

$$
\mathbb{Q}\left[\phi\left(\frac{\tau}{n}\right) \phi\left(\frac{\tau+1}{n}\right) \ldots \phi\left(\frac{\tau+n-1}{n}\right)\right] .
$$

4. Approach to the Weierstrass $\wp$-function and some modular functions. Let us consider $\wp(\tau / 2)$ for $\tau / 2 \notin \Lambda_{\tau}$ where $\Lambda_{\tau}$ is the lattice $\mathbb{Z}+\tau \mathbb{Z}$. Let $r, v$ be positive odd integers and $s, u$ any positive integers such that $(r, s)=(u, v)=1$. We then see that $\frac{r}{2 s} \tau, \frac{v}{2 u} \tau$ are imaginary quadratic and $\frac{r}{2 s} \tau, \frac{v}{2 u} \tau \notin \Lambda_{\tau}$. By Proposition 2.1 and Theorem 2.2,

$$
\frac{\wp\left(\frac{r}{2 s} \tau\right)}{\wp\left(\frac{v}{2 u} \tau\right)}=\frac{\wp\left(\frac{r}{2 s} \tau\right)}{\pi^{2} \eta\left(\frac{r}{s} \tau\right)^{4}} \cdot \frac{\pi^{2} \eta\left(\frac{v}{u} \tau\right)^{4}}{\wp\left(\frac{v}{2 u} \tau\right)} \cdot \frac{\eta\left(\frac{r}{s} \tau\right)^{4}}{\eta(\tau)^{4}} \cdot \frac{\eta(\tau)^{4}}{\eta\left(\frac{v}{u} \tau\right)^{4}}
$$

is an algebraic number. Thus we get the following

THEOREM 4.1.

$$
\frac{\wp\left(\frac{r}{2 s} \tau\right)}{\wp\left(\frac{v}{2 u} \tau\right)}
$$

is an algebraic number, where $\tau, r, s, u$ and $v$ are defined as above.

Let

$$
f_{0}(z ; \tau)=-2^{7} 3^{5} \frac{g_{2}(\tau) g_{3}(\tau)}{\Delta(\tau)} \wp\left(z, \Lambda_{\tau}\right)
$$

be the first Weber function for $\tau \in \mathfrak{h}$ and $z \in k$. Having fixed the integer $N>1$, for $r, s$ in $\mathbb{Z}$ not both divisible by $N$, let

$$
f_{r, s}(\tau)=f_{0}\left(\frac{r \tau+s}{N} ; \tau\right)
$$

be the Fricke function.

THEOREM 4.2. Let $\tau \in \mathfrak{h} \cap k$ and $r, s$ be positive integers not both divisible by $N$ for fixed integer $N>1$. Then every element $g(\tau)$ in $\Omega\left(j(\tau), f_{r, s}(\tau)\right)$ is an algebraic number, where $\Omega$ is a field of algebraic numbers. In particular, any $g(\tau) \in \mathbb{Z}\left[j(\tau), 32 r^{2} f_{r, s}(\tau)\right]$ is an algebraic integer. 
Proof. We can derive that

$$
\begin{aligned}
f_{r, s}(\tau) & =f_{0}\left(\frac{r \tau+s}{N} ; \tau\right) \\
& =-2^{7} 3^{5} \frac{g_{2}(\tau) g_{3}(\tau)}{\Delta(\tau)} \wp\left(\frac{r \tau+s}{N} ; \tau\right) \\
& =-\frac{1}{8} \cdot \frac{3 g_{2}(\tau)}{4 \pi^{4} \eta(\tau)^{8}} \cdot \frac{27 g_{3}(\tau)}{\pi^{6} \eta(\tau)^{12}} \cdot \frac{3 \wp\left(\frac{r \tau+s}{N}\right)}{\pi^{2} \eta\left(\frac{2 r \tau+2 s}{N}\right)^{4}} \cdot \frac{\eta\left(\frac{2 r \tau+2 s}{N}\right)^{4}}{\eta(\tau)^{4}} .
\end{aligned}
$$

Put $\alpha_{2 r / N}=\left(\begin{array}{cc}2 r & 2 s \\ 0 & N\end{array}\right)$ with $2 s \bmod N$. It follows from Proposition 2.1 that

$$
\phi_{\alpha_{2 r / N}}(\tau)^{1 / 6}=\left(\frac{1}{N^{12}}|2 r N|^{12} \frac{\eta\left(\frac{2 r \tau+2 s}{N}\right)^{24}}{\eta(\tau)^{24}}\right)^{1 / 6}=(2 r)^{2} \frac{\eta\left(\frac{2 r \tau+2 s}{N}\right)^{4}}{\eta(\tau)^{4}}
$$

is an algebraic integer.

We then conclude by Theorem 2.2 that $32 r^{2} f_{r, s}(\tau)$ is an algebraic integer. Therefore the theorem follows.

\section{References}

[1] B. C. Berndt, H. H. Chan and L.-C. Zhang, Ramanujan's remarkable product of theta-functions, Proc. Edinburgh Math. Soc. 40 (1997), 583-612.

[2] K. Chandrasekharan, Elliptic Functions, Grundlehren Math. Wiss. 281, Springer, 1985.

[3] M. Deuring, Die Typen der Multiplikatorenringe elliptischer Funktionenkörper, Abh. Math. Sem. Univ. Hamburg 14 (1941), 197-272.

[4] S. Lang, Elliptic Functions, Addison-Wesley, 1973.

[5] A. Néron, Modèles minimaux des variétés abéliennes sur les corps locaux et globaux, Inst. Hautes Études Sci. Publ. Math. 21 (1964), 5-128.

[6] J.-P. Serre and J. Tate, Good reduction of abelian varieties, Ann. of Math. 88 (1968), 492-517.

[7] G. Shimura, Introduction to the Arithmetic Theory of Automorphic Forms, Princeton Univ. Press, Princeton, NJ, 1971.

[8] J. H. Silverman, Advanced Topics in the Arithmetic of Elliptic Curves, Springer, New York, 1994.

[9] —, The Arithmetic of Elliptic Curves, Springer, New York, 1986.

[10] E. T. Whittaker and G. N. Watson, A Course of Modern Analysis, Cambridge Univ. Press, 1978.

Department of Mathematics Chonbuk National University Chonju 561-756, South Korea E-mail: dykim@math.chonbuk.ac.kr
Department of Mathematics Korea Advanced Institute of Science and Technology Taejon 305-701, South Korea 